\title{
An Analysis of Underground Forums
}

\author{
Marti Motoyama, Damon McCoy, Kirill Levchenko, Stefan Savage and Geoffrey M. Voelker \\ Department of Computer Science and Engineering \\ University of California, San Diego
}

\begin{abstract}
Underground forums, where participants exchange information on abusive tactics and engage in the sale of illegal goods and services, are a form of online social network (OSN). However, unlike traditional OSNs such as Facebook, in underground forums the pattern of communications does not simply encode pre-existing social relationships, but instead captures the dynamic trust relationships forged between mutually distrustful parties. In this paper, we empirically characterize six different underground forums BlackHatWorld, Carders, HackSector, HackElite, Freehack, and L33tCrew - examining the properties of the social networks formed within, the content of the goods and services being exchanged, and lastly, how individuals gain and lose trust in this setting.
\end{abstract}

\section{Categories and Subject Descriptors}

H.3.5 [Information Storage and Retrieval]: Online Information Services; J.4 [Computer Applications]: Social and Behavioral Sciences; K.4.4 [Computers and Society]: Electronic Commerce

\section{General Terms}

Human factors, Measurement, Security

\section{Keywords}

Underground forums, Online social networks

\section{INTRODUCTION}

Online social networks (OSNs) capture, in a concrete form, the character and dynamics of human social relationships. Consequently, the popularity of such services (e.g., Facebook, Twitter, etc.) has been followed closely by researchers using the explicit nature of these networks to characterize social graph properties and how they inform user interaction $[6,11]$. While less well explored, there are also a range of implicit social networks defined via interaction on other shared interaction sites (e.g., Web forums, blogs, etc.). In this paper, we focus on a particular sub-population of such activities: underground forums.

Users of underground forums participate in many activities similar to those found on traditional online social networks: they maintain profiles, add fellow users to buddy lists, and engage in conversations via private messaging. However, the "raison d'etre" for

Permission to make digital or hard copies of all or part of this work for personal or classroom use is granted without fee provided that copies are not made or distributed for profit or commercial advantage and that copies bear this notice and the full citation on the first page. To copy otherwise, to republish, to post on servers or to redistribute to lists, requires prior specific permission and/or a fee.

IMC'11, November 2-4, 2011, Berlin, Germany.

Copyright 2011 ACM 978-1-4503-1013-0/11/11 ...\$10.00. such forums is not simply for social contact, but to support criminal (or at best "grey hat") activities. Thus, users of these forums regularly engage in the buying, selling and trading of abusive services and illegally obtained goods such as credit card numbers, online currencies, compromised accounts and even drugs. However, since underground users frequently only know each other online (and via pseudonyms even there), they must develop new means to establish trust among themselves.

In this paper, we examine these implicit social networks and how they are used in the context of six underground forums BlackHatWorld, Carders, FreeHack, HackEl1te, HackSector, and L33tCrew - for which we have complete activity records. Our analysis is organized into three parts: first, we analyze the structure of the underlying social networks present on the forums, followed by an examination of the commercial aspects of the sites (e.g., what types of products are being sold, who are the most active players in the market, etc.) and finally we look at how different reputational factors impact behavior. We believe our work is the first analysis of this type and provides valuable insight into how online criminal actors create and develop social relationships in support of their goals.

\section{BACKGROUND}

Online underground markets have existed in various forms for decades. Early markets used Internet Relay Chat (IRC), documented by Thomas et al. [10] and Franklin et al. [4], to provide a public medium for sharing information about the availability and pricing of goods and services (e.g., stolen credit cards, accounts, botnets, cash out services, etc.). ${ }^{1}$ Over time, many of these markets moved to using persistent Web forums and expanded to cover a broader range of information sharing. Zhuge et al. first documented the use of such forums in China [12] and contemporary analyses have been published by Holt et al. [5], Radianti [7] and Fallmann et al. [3]. Over time, some of these forums have specialized and many have moved to "closed" models (i.e., in which new members must be explicitly vouched for by existing members); for example, StoneGross et al. [9] recently documented the membership and goods on offer on the private Spamdot.biz forum, which specialized in support for email spammers. Ultimately, the goal of all such forums is to expand the knowledge base of the participants (e.g., which registrars will "look the other way", how to best manipulate Google ranking results, etc.) as well as to expand the set of potential trading partners. However, there is little public research that empirically examines the social networks formed in such forums or the mechanisms employed to manage trust. Indeed, such analyses can be difficult since modern forums combine public sections, restricted sections (requiring higher status) and person-to-person private messages (PMs) that may not be externally visible.

\footnotetext{
${ }^{1}$ For a brief overview of the how this credentials market operates today, see Shilman [8].
} 


\begin{tabular}{|c|c|c|c|c|c|c|c|c|}
\hline Forum & Abbrv & Dates Covered & Subforums & Threads & Posts & Pvt Msgs & Users & Lurkers \\
\hline L33tCrew & $\mathrm{LC}^{*}$ & May 07 - Nov 09 (30 mo.) & 239 & 120,560 & 861,459 & 501,915 & 18,834 & $46.5 \%$ \\
\hline HackSector & HS & Nov 01 - Nov 07 (72 mo.) & 147 & 72,734 & 724,820 & 78,777 & 33,986 & $55.0 \%$ \\
\hline FreeHack & FH & Jul 04 - Dec 10 (77 mo.) & 152 & 62,972 & 499,736 & 112,318 & 38,377 & $62.9 \%$ \\
\hline Carders & $\mathrm{CC}^{*}$ & Sep 08 - Dec 10 (27 mo.) & 121 & 52,188 & 373,143 & 197,067 & 8,425 & $35.0 \%$ \\
\hline BlackHatWorld & $\mathrm{BH}$ & Oct 05 - Mar 08 (29 mo.) & 38 & 7,270 & 65,572 & 20,849 & 8,718 & $47.9 \%$ \\
\hline HackeL1te & HL & Mar 10 - Apr 11 (13 mo.) & 43 & 5,501 & 9,018 & 541 & 2,431 & $66.7 \%$ \\
\hline
\end{tabular}

Table 1: Summary of the data from the six forums, ranked by number of posts $(*$ denotes forums geared toward commerce).

\begin{tabular}{l|rrrr|rrrr|rrrr}
\hline & \multicolumn{4}{|c}{ Buddy } & \multicolumn{4}{c}{ Private Message } & \multicolumn{4}{c}{ Thread } \\
Forum & Partic. & Links & L/P & WCC & Partic. & Links & L/P & WCC & Partic. & Links & L/P & WCC \\
\hline LC & 2,587 & 4,448 & 1.7 & 214 & 7,898 & 170,954 & 21.6 & 7 & 9,124 & $3,791,330$ & 415.5 & 1 \\
HS & 1,282 & 1,057 & 0.8 & 352 & 9,562 & 43,807 & 4.6 & 69 & 13,502 & $2,582,513$ & 191.3 & 1 \\
FH & 1,921 & 5,944 & 3.1 & 100 & 10,294 & 55,945 & 5.4 & 21 & 11,833 & $1,473,824$ & 124.6 & 3 \\
CC & 1,000 & 1,834 & 1.8 & 154 & 5,065 & 63,409 & 12.5 & 12 & 4,593 & 792,962 & 172.6 & 2 \\
BH & 199 & 205 & 1.0 & 37 & 3,438 & 11,183 & 3.3 & 3 & 2,940 & 320,028 & 108.9 & 2 \\
HL & 38 & 65 & 1.7 & 4 & 174 & 271 & 1.6 & 5 & 690 & 10,477 & 15.2 & 3 \\
\hline
\end{tabular}

Table 2: Summary of the basic social networking statistics for each forum. Partic. means participants, or users who have links of the specified type; $L / P$ represents the number of links divided by the number of participants. WCC means weakly connected components.

\section{DATA OVERVIEW}

In this study we have the luxury of "ground truth" - complete records of six underground forums via SQL dumps of their underlying databases. We do not claim that these six are representative of all underground forums, but they provide us with a starting point for understanding the dynamics of underground forums. Each of these datasets has been acquired by unknown outside parties and made public ("leaked") via various methods. Each forum contains a wealth of information: user registration data, private messages exchanged, forum posts, member status changes, banned user logs, etc. For a more comprehensive list of the available data, please refer to the Invision Power Board (for L33tCrew) and vBulletin database schemas $[1,2]$. We briefly describe the purpose of each forum.

BlackhatWorld (BH) was founded in approximately 2005 and is primarily English speaking. The main focus of $\mathrm{BH}$ is blackhat search engine optimization (SEO), a practice in which users attempt to abusively manipulate search engine algorithms to gain increased page rank. At the time our dataset was obtained, BH did not have a vibrant trading marketplace, as the site was initially oriented towards the discussion of blackhat techniques. Today, however, the site contains over 800 threads in the services-for-sale section and more than 275 threads in the goods-for-sale section.

Carders (CC) is a German-speaking site primarily focused on the monetization of stolen credit card numbers and bank account information. The site is heavily geared towards the exchange of goods and services. The L33tCrew (LC) forum is very similar to $\mathrm{CC}$, both in its content and the types of products exchanged.

Freehack (FH) is another German site, but does not target any one industry. The threads on the forum cover a number of different topics, ranging from crypting (encoding software to make detecting malware more difficult) to video games. Items for sale include Steam (gaming) accounts, automatic account creators, and hacking software. The users typically do not buy or sell stolen credentials. The remaining sites, HackSector (HS) and HackeL1te (HL), are similar although HL is English speaking.

Table 1 summarizes the membership and activity across each of these forums. In total, our analysis covers over 2.5 million posts, $900 \mathrm{k}$ private messages, and $100 \mathrm{k}$ users. Our dataset also spans a range of time periods, with the FH and HS datasets covering approximately six years, while HL is our shortest at roughly a year. LC is our largest dataset by forum activity, with the largest number of threads (120k), posts (860k), and private messages $(500 \mathrm{k})$. The six forums exhibit different properties with regards to the number of posts and private messages exchanged. The forums geared towards commerce, $\mathrm{CC}$ and LC, have a much higher number of private messages, since many business transactions occur over private messaging. For example, we observed 23-26 PMs/user for CC and LC, while the other four forums, whose users primarily swap information, exhibit less than 3 PMs/user. Across all forums a fair number of "lurkers", or individuals who simply register an account but take no action, exist on each forum. Over 55\% of the users on FH, HS and HL are lurkers. Again, the trading forums have a smaller fraction of lurkers with only $35 \%$ in CC and $46 \%$ in LC.

\section{UNDERGROUND SOCIAL NETWORKS}

In this section, we analyze the structural properties of the six different forums. This task is nontrivial in the context of a forum, since the definition of a link between nodes (users) remains ambiguous. To that end, we consider three types of relationships that exist in the forums: buddy, private message, and thread. Table 2 summarizes the basic social networking statistics for each forum.

A buddy link is the most explicit relationship that exists between users, and is a directed link since buddy requests may be accepted, pending, or denied. Thus, accepted requests result in symmetric links, while pending requests produce unidirectional links. However, the number of explicitly declared buddy relationships across the forums is quite small. Less than $10 \%$ of all users in each forum issued a friend request to another user, suggesting that underground forum users do not think of their relationships as persistent, or that the members see no utility in friending other users.

Because buddy links do not fully capture the latent relationships present in the forums, we further analyze the social network by including links that result from private messaging. If user $u_{1}$ sends a PM to user $u_{2}$, we establish a directed link from $u_{1}$ to $u_{2}$.

Lastly, thread relationships result when two users post in the same sub-forum thread. To establish these links, we order all posts in the same thread by their post times. We then create a link from user $u_{2}$ to user $u_{1}$ if $u_{2}$ posted after $u_{1}$, with the reasoning being that $u_{2}$ is interacting with all users in the thread prior to his or her post. Unsurprisingly, Table 2 shows that these one-to-many thread relationships produce the most links and fewest weakly connected components. One may use more advanced techniques (e.g., parsing "[QUOTE]" and "@<username>" expressions) to establish finergrained thread relationships, but we leave this to future work. 


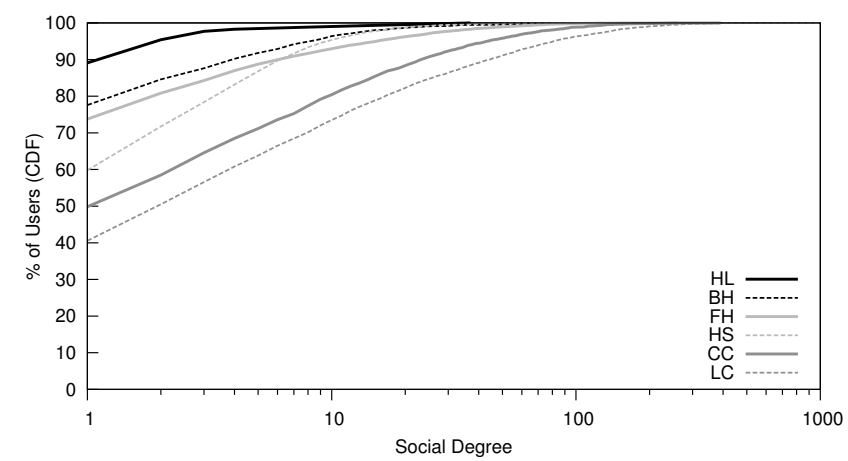

(a) Private Message

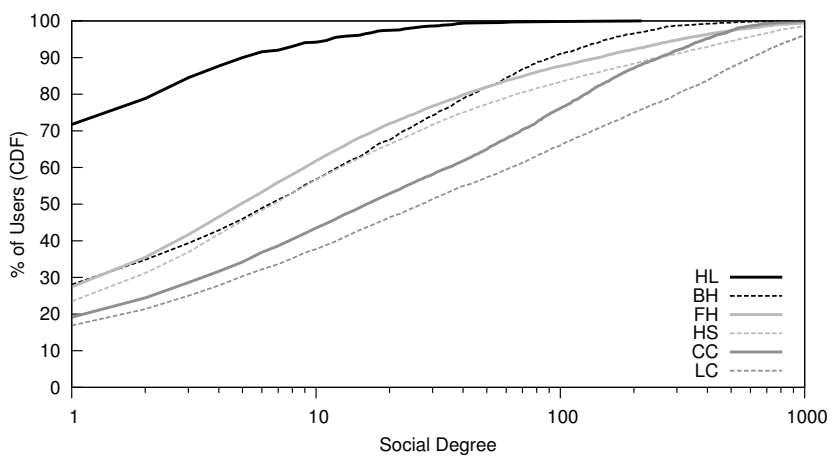

(b) Thread

Figure 1: Degree distribution for reciprocated links.

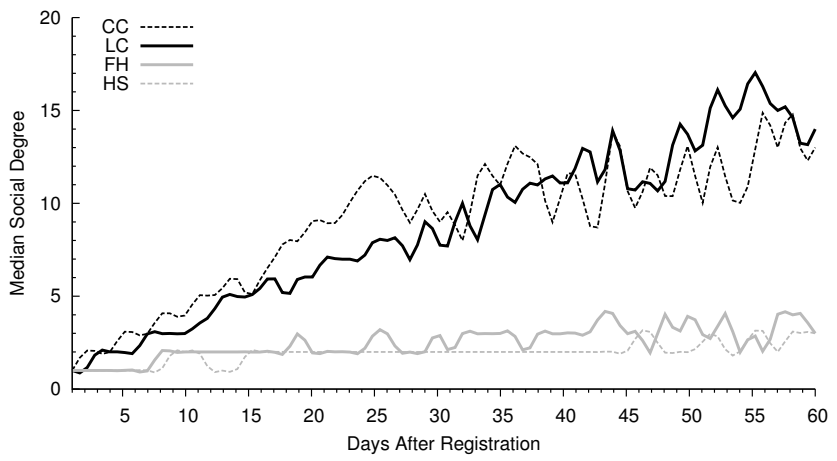

Figure 2: The median social degree for users based on private messages in a 60-day window surrounding their registration dates.

Because so few buddy links exist, we only consider the private message ("PM") and thread ("thread") link types in our analyses.

\subsection{Degree Analysis}

Figure 1 shows the degree distributions for the six forums. We only consider pairs of nodes with reciprocal links. Figure 1(a) shows that few users exchange private messages on $\mathrm{HL}, \mathrm{BH}, \mathrm{FH}$ and $\mathrm{HS}$, with less than $40 \%$ of users involved in more than one private message exchange. Members on CC and LC create more relationships through private messages, with over $50 \%$ of their users linked to at least one other forum member, and more than $25 \%$ of their users involved in at least 10 conversations. This difference is likely due to users being more actively engaged in commerce, with many transactions taking place over private messages. The users in all forums (except for HL, being relatively new) create a comparable number of links via posting, which makes sense since this is the primary means of communication in forums. CC and LC exhibit more links, with over $50 \%$ of their users linked to over 20 other members via public postings (double the amount of other forums).

\subsection{Social Network Growth}

Figure 2 shows how the median private messaging social degree changes among forum users in the 60 days following their registrations. We only consider reciprocal links when computing the social degree. For each pair of linked users, we looked at the difference between their registration dates and the latest timestamp associated with their first private message exchange. We omit HL and BH due to the noise present in their curves. For the private messaging social graph, there is little change in the median social degree after the first week for users on FH and HS. In contrast, for CC and LC, both sets of users experience growth after the first week, again likely due to the business transactions occurring over PM to a dynamic set of

\begin{tabular}{rr|rrr|rr}
\hline & & \multicolumn{3}{|c|}{ German $(D E)$} & \multicolumn{2}{|c}{ English $(E N)$} \\
& & LC & FH & CC & BH & HL \\
\hline \multirow{3}{*}{$D E$} & LC & - & 13.99 & 3.16 & 0.05 & 0.03 \\
& FH & 6.87 & - & 2.23 & 0.02 & 0.08 \\
& CC & 7.09 & 10.19 & - & 0.02 & 0.39 \\
\hline \multirow{2}{*}{$E N$} & BH & 0.11 & 0.10 & 0.02 & - & 0.00 \\
& HL & 0.25 & 1.19 & 1.36 & 0.00 & - \\
\hline
\end{tabular}

Table 3: Percentage of overlapping email addresses across all pairs of forums. Percentages listed in each row are with respect to the population size of the forum specified in that row. The HS dataset lacked addresses.

\begin{tabular}{|c|c|c|c|c|c|c|c|}
\hline & \multicolumn{4}{|c|}{ German $(D E)$} & \multicolumn{2}{|c|}{ English $(E N)$} \\
\hline & & $\mathbf{L C}$ & HS & FH & $\mathrm{CC}$ & BH & HL \\
\hline \multirow{4}{*}{$D E$} & $\mathrm{LC}$ & - & 17.34 & 21.47 & 6.18 & 1.57 & 0.62 \\
\hline & HS & 9.61 & - & 13.13 & 3.03 & 1.24 & 0.44 \\
\hline & $\mathrm{FH}$ & 10.54 & 11.63 & - & 4.43 & 1.07 & 0.46 \\
\hline & $\mathrm{CC}$ & 13.80 & 12.21 & 20.19 & - & 1.86 & 1.03 \\
\hline \multirow{2}{*}{$E N$} & $\mathrm{BH}$ & 3.38 & 4.82 & 4.73 & 1.80 & - & 0.33 \\
\hline & $\mathrm{HL}$ & 4.77 & 6.17 & 7.20 & 3.58 & 1.19 & - \\
\hline
\end{tabular}

Table 4: Percentage of overlapping usernames across all pairs of forums.

partners. After the first month, the median social degree for the users on $\mathrm{LC}$ and $\mathrm{CC}$ is approximately nine, versus two for $\mathrm{FH}$ and HS. The growth rate for the thread social graph (not shown) follows the same trend across all forums: users undergo the largest growth in the first several weeks. In contrast, however, all forums show members continuing to interact with new users via public postings.

\subsection{User Overlap}

To study the population overlap among forum members, Tables 3 and 4 show the percentage of overlapping email addresses and usernames (respectively) between each pair of forums. Of the six, only HS did not list any email addresses for its registered members. LC and FH share over two thousand email addresses, while LC and CC share 595 , roughly $7 \%$ of the CC membership. This overlap is unsurprising, since both forums are geared toward the trading of stolen credit cards. However, even the newest English-based forum, HL, shared 6 to 33 email addresses across the German forums. Though email addresses are likely a more reliable metric for establishing population overlap, usernames also provide insight into overlap since users desire unique public identities to maintain their reputations. All forum pairs contained some number of overlapping usernames, with the German forums (CC, FH, HS, LC) sharing an appreciable number of usernames. For example, over $10 \%$ of the usernames on CC are present in all three of the other German forums. Likewise, over $17 \%$ of the usernames on LC appear in both FH and HS. 


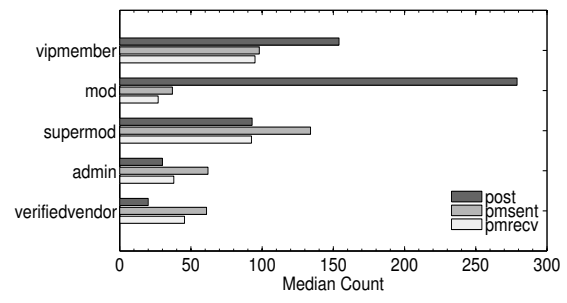

Figure 3: Median activity users engaged in prior to transitioning groups for CC.

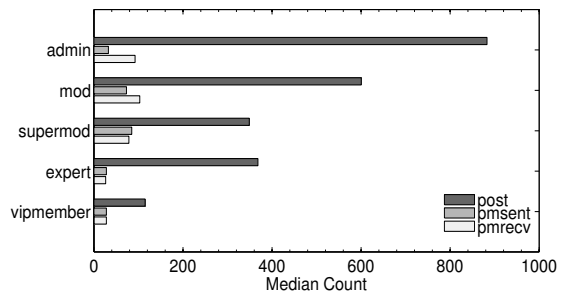

Figure 4: Median activity users engaged in prior to transitioning groups for $\mathrm{FH}$.

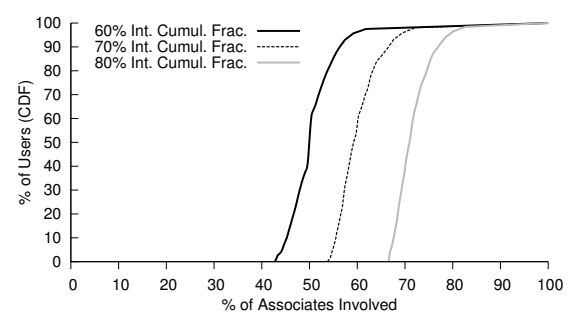

Figure 5: Distribution of users' interactions for PMs on LC.

\begin{tabular}{l|rr|rr|l}
\hline \multirow{2}{*}{ Category } & \multicolumn{2}{|c|}{ Threads } & \multicolumn{2}{c|}{ Users } & Top \\
& B & \multicolumn{1}{c}{ S } & B & S & Subcategory \\
\hline payments & 5,294 & 5,074 & 1,354 & 1,281 & paysafecard \\
game-related & 935 & 951 & 449 & 459 & steam \\
credit cards & 597 & 798 & 339 & 421 & unspecified cc \\
accounts & 761 & 566 & 382 & 356 & ebay \\
merchandise & 390 & 518 & 246 & 334 & iphone \\
software/keys & 355 & 485 & 214 & 296 & key/serial \\
services & 155 & 562 & 119 & 384 & carder \\
victim logs & 380 & 334 & 237 & 232 & viclog \\
mail/drop srvs & 347 & 292 & 248 & 203 & packstation \\
fraud tools & 203 & 343 & 132 & 239 & socks \\
\hline
\end{tabular}

Table 5: Top 10 most commonly traded merchandise categories on CC

\subsection{Group Elevation}

Users that join a forum are assigned a group, which roughly corresponds to their social status on the site. Generally, users start in the pending authorization group, meaning they must perform some action (e.g., respond to email confirmation) or undergo some type of scrutiny before being given access to the forum. Once the user has jumped through the necessary hoops, they begin in the "newbie" group. After some activity, users are generally elevated to a non-newbie group and advance from there. Figures 3 and 4 show the median amount of activity that users engaged in prior to transitioning to higher group levels for $\mathrm{CC}$ and $\mathrm{FH}$ (BH and $\mathrm{HL}$ were similar to FH). All the forums place a large emphasis on public postings versus private messaging, indicating that reputation comes from being publicly active on the forums. Users with greater standing in the $\mathrm{CC}$ forum have the most balanced amount of activity, posting and private messaging in roughly equal amounts.

\subsection{User Interaction Analysis}

Figure 5 shows how private message interactions are distributed among users' "associates" (i.e., fellow members they are linked with) on LC, which has the greatest number of PMs. We looked at these distributions to determine the extent to which users interact with different individuals. For each user, we compute a distribution of private messaging events over the user's associates. We then looked at the $60 \%, 70 \%$, and $80 \%$ points in that distribution. Figure 5 suggests that users on LC exchange private messages with a diverse set of individuals, versus users on traditional OSNs, who interact with few of their friends. Wilson et al. [11] found that, for users on Facebook, 20\% of their friends account for $70 \%$ of their interactions. In contrast, for users on LC, approximately $70 \%$ of their associates are responsible for $70 \%$ of their private messages. The corresponding graph for users linked via threads is similar.

\section{MARKETPLACE}

In this section we look at the types of goods and services exchanged on LC and CC, the two forums with the most well devel-

\begin{tabular}{l|rr|rr|l}
\hline \multirow{2}{*}{ Category } & \multicolumn{2}{|c|}{ Threads } & \multicolumn{2}{c|}{ Users } & Top \\
& B & \multicolumn{1}{c}{ S } & B & \multicolumn{1}{c}{ S } & Subcategory \\
\hline payments & 8,507 & 8,092 & 1,539 & 1,409 & paysafecard \\
game-related & 2,379 & 2,584 & 924 & 987 & steam \\
accounts & 2,119 & 2,067 & 850 & 974 & rapidshare \\
credit cards & 996 & 1160 & 467 & 566 & unspecified cc \\
software/keys & 729 & 1410 & 422 & 740 & key/serial \\
fraud tools & 652 & 1155 & 363 & 601 & socks \\
tutorials/guides & 950 & 537 & 562 & 393 & tutorials \\
mail/drop srvs & 751 & 681 & 407 & 364 & packstation \\
merchandise & 493 & 721 & 264 & 404 & ipod \\
services & 266 & 916 & 176 & 555 & carder \\
\hline
\end{tabular}

Table 6: Top 10 most commonly traded merchandise categories on LC.

oped and active trading marketplaces. We first look at what types of goods are traded among these two underground communities, and then analyze how social degree and reputation affect trading.

\subsection{Merchandise}

To determine what types of items are available on the forums, we extracted thread titles containing the markers "[B]" or " $[\mathrm{S}]$ ", denoting items that are being traded for and sought after, respectively. We then wrote over 500 regular expressions to bin the items into 18 categories; these hand-defined categories include merchandise, banking information, drugs, mailing and dropping services, and a number of other commonly observed wares/services. We created the categories based on domain knowledge of illicit goods and by randomly sampling trading thread titles. Using our regular expressions, we categorized $87 \%$ of the $14,430 \mathrm{CC}$ threads and $77 \%$ of 31,923 LC threads. Because users typically list several items for trade in a single thread, a thread may be counted in multiple categories. There is a long tail of merchandise types that we did not cover with our regular expressions; for example, on LC, threads mention such items as "Internet hack N95" or "Proteine - Inko XTREME Muscle Gainer", while on CC, threads offer up such goods as "Conrad.de Kundenlogins" or "Pall Mall umsonst".

Tables 5 and 6 show the top 10 most commonly traded items on CC and LC (respectively), ordered by the number of total binned threads in the designated category. The thread column shows the number of thread titles containing terms associated with the category, while the user column shows the number of distinct users who created those threads. The "B" and " $\mathrm{S}$ " columns denote threads where items were being traded for or sought after, respectively.

The items most commonly traded for are offline/online payments, including PayPal, cash, Ukash, and PaySafeCards (PSC). Over 5\% of all threads involve trading for offline/online payments on both forums. Traders in the underground market prefer PSC, a type of prepaid online currency that is widely used in Europe. Gaming accounts, in particular Steam, are the second most commonly traded item; credit cards and accounts make up the next two traded for 


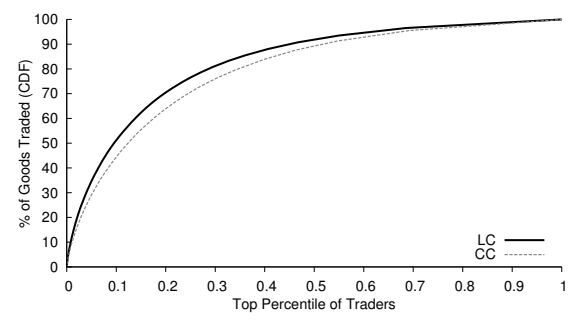

Figure 6: Top percentile of traders vs percentage of goods traded.

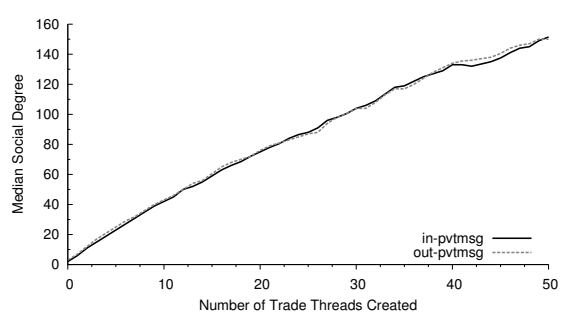

Figure 7: Number of LC trading threads created vs. median social degree.

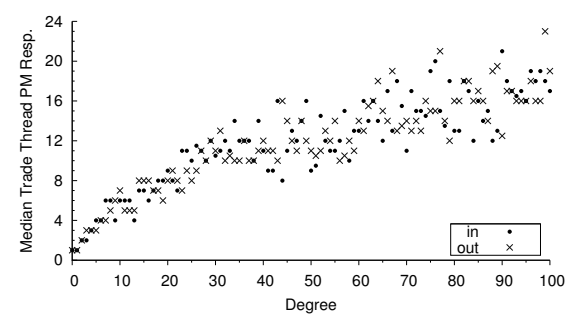

Figure 8: PM social graph degree vs median number of trading thread response PMs for LC. items. While not shown in the table, drugs also made up a notable number of traded items on CC: over 100 threads listed weed, 25 mentioned Viagra, and 15 specified speed.

Next, we look at the number of trading threads the top merchants are responsible for. Figure 6 shows the relationship between the top percentile of traders on both forums and the percentage of goods traded. For example, the top $10 \%$ of the traders on both sites (measured by the number of trading threads created by the user) account for $40-50 \%$ of the goods traded. One implication of this trading distribution is that law enforcement can pursue the top tier traders to shut down much of the marketplace activity. The top traders can create multiple accounts to mask their activity levels, but accruing reputation for possibly numerous accounts is nontrivial.

\subsection{How Social Degree Affects Trading}

Now, we analyze the effect of the social network on trading. Figure 7 shows how the median social degree measured in private message links increases for users after posting trading threads on LC. We order each user's trading threads by time. Subsequently, we compute the user's PM social degree just before he or she creates each thread. We then bin that social degree with respect to the thread's posting order, taking the median over all users. The results suggest that, as users trade more, they continue to interact with increasingly larger numbers of individuals and hence, potential customers. For LC, the median out and in degrees for the PM social graphs increases at a roughly constant rate of four for every trading thread created. The CC graph looks similar; the PM out/in degree increases at a rate between one and two for each thread.

We now investigate how PM in/out degree affects the response to a trading thread. Figure 8 plots the in/out PM degrees for LC users before they post a trading thread against the number of PMs they receive in the following week. In comparison, LC and CC members receive on average 0.07 (standard deviation $\sigma=0.68$ ) and $0.11(\sigma=1.19)$ private messages per week when issuing no posts prior to that week. The graph suggests that traders with higher PM social graph degrees receive more PMs in the week after they post a trading thread. For CC, the numbers look similar, but become increasingly noisier after an in/out degree of 30 .

Lastly, we look at what fraction of private messages are sent by new users to traders in the week following a trading thread post. Figure 9 shows that for approximately $30 \%$ of all trading threads, the posters receive only PMs from individuals they have interacted with before. For $50 \%$ of the trading threads on LC and CC, 60-75\% of the PMs came from prior acquaintances. Finally, for approximately $20-25 \%$ of all trading threads, the trader interacted with only new people.

\subsection{Effect of Group Status}

Figure 10 shows how a user's group affects the responsiveness to a trading thread; we focus on CC because LC does not contain any data regarding user group transitions. We looked at the trading threads posted by users at a certain group level. We then determined how many PMs the users received in the week after posting trading threads. Figure 10 suggests that a user's group status does influence how many PMs the user receives upon posting a trading thread. Verified vendors and VIP members receive between 2-3 times more response PMs than "newbies".

\subsection{Effect of Ratings}

The only forum with an explicit rating system for trading transactions is CC. The rating system is ternary: traders receive either a positive, negative, or neutral feedback. Surprisingly, most of the reviews are positive: of the 3,157 reviews ( $20 \%$ of discernible trading threads), only 67 were negative and 2 were neutral. Of the 67 negatively rated users, 43 were banned, and the rest were generally rated negatively due to "poor" or "unfriendly" service. We suspect that bad traders are outed publicly in separate thread posts (see Section 6.2) and banned before being rated. Because so few users were rated poorly, we do not differentiate between the rating types.

We now consider the effect of the rating system on the amount of interest a trader receives (either in the form of response posts or private messages) after posting a trading thread. Figure 11 shows the effects of the first 10 ratings on the median number of PMs users receive in the week following a trading thread post. With zero ratings, the median response $\mathrm{PM}$ count is one, but with a single rating, the median count rises to 15 . The median PM count continues to increase from there, though not in a well-defined manner. While rated traders receive more private messages, they do not experience an increase in the number of response posts; the median response post count remains constant at one. We speculate that the rating system lends more credibility to a trader's threads, and people are not so quick to question the trader's reputation in the public space.

\subsection{Activity To First Rating}

We now analyze how much activity users must participate in before they earn enough trust such that they engage in a business transaction with another forum member. We measure the number of actions users take (postings, private messaging) before they receive their first ratings. Relying on the rating system is subject to error, since users can conduct business outside of the forum, but it provides us with some idea about how trust is earned. Figure 12 shows that, before approximately $50 \%$ of users received their first ratings, they posted around 60 times in 50 different threads, received about 35 private messages from 13 users, and sent around 33 private messages to 13 users.

\section{BANNED ANALYSIS}

Some users in underground forums behave maliciously towards other members. In this section, we look at the top reasons why users are banned from the forums. We also investigate different properties associated with accusations of fraud in the marketplace. 


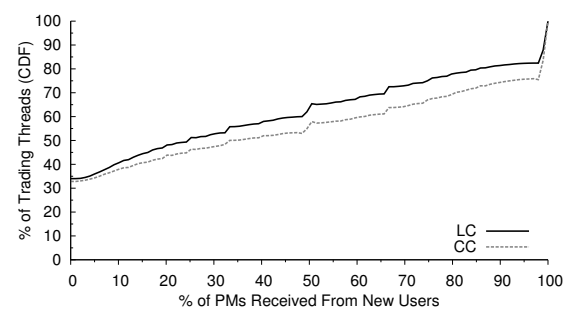

Figure 9: Fraction of PMs received from new users after posting a trading thread.

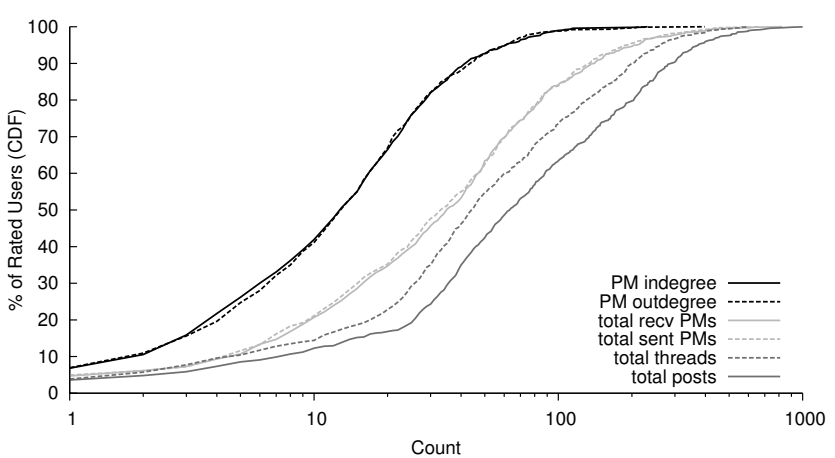

Figure 12: CDF showing the activities a user participated in prior to receiving a first rating.

\begin{tabular}{l|lr|lr|lr}
\hline & \multicolumn{2}{|c|}{ Rank 1 } & \multicolumn{2}{|c|}{ Rank 2 } & \multicolumn{2}{c}{ Rank 3 } \\
Forum & Reason & $\%$ & Reason & $\%$ & Reason & $\%$ \\
\hline BW (22) & spammer & 40.9 & dup. acc & 22.7 & infl. posts & 22.7 \\
CC $(1,587)$ & dup. acc & 60.7 & ripper & 12.1 & spammer & 7.3 \\
FH (447) & dup. acc & 30.6 & malware & 16.6 & spammer & 14.1 \\
HS (317) & spammer & 24.6 & malware & 10.7 & dup. acc & 9.8 \\
HL (75) & infl. posts & 52.0 & trade-rel. & 34.7 & spammer & 13.3 \\
LC (247) & misuse & 37.7 & spammer & 17.4 & ripper & 17.0 \\
\hline
\end{tabular}

Table 7: The top three reasons why users are banned on each of the forums.

\subsection{Why Users are Banned}

The fraction of users that are banned on the forums is non-trivial. For example, the moderators on CC have banned over $20 \%$ of the users that appear in their members table. Because the individuals who participate in underground forums generally lack some scruples, this is not surprising. Table 7 shows the top three reasons why users are banned in the forums. To generate the data, we analyzed a specific table in five of the forums (BW, CC, FH, HS, HL) that explicitly holds information regarding user bans. LC did not have such a table, so we analyzed the warnings issued to banned users and assumed that the final warning received by the user resulted in the ban. Next, we created 13 categories for the most commonly appearing banned reasons and wrote 35 regular expressions to bin them. Again, users can be banned for multiple reasons. Also, not every ban is explained (the reason is sometimes left blank), so the percentages shown are with respect to the explained bans (represented by the numbers in parenthesis). These regular expressions covered over $70 \%$ of the banned reasons across all five forums.

The most common problem across the forums involves duplicate accounts (dup. acc), which appears in the top three reasons in four of the forums. Users often create duplicate accounts to circumvent a prior ban. Another problem in the forums is, ironically, spamming and malware attacks, with BW and HS particularly inundated with spammers. In the commerce oriented forums (LC and CC), rippers

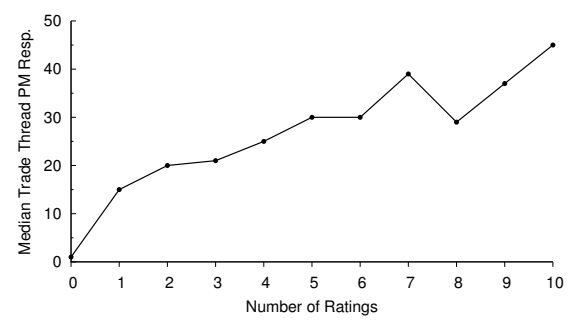

Figure 11: Effect of ratings on response to trading threads.

\begin{tabular}{l|rr|rr}
\hline & \multicolumn{2}{|c|}{ Banned } & \multicolumn{2}{c}{ Unbanned } \\
Stat Name & Accuser & Accused & Accuser & Accused \\
\hline Num PMs Sent & 243.0 & 34.5 & 22.0 & 73.5 \\
Num PMs Recv & 271.0 & 31.0 & 24.5 & 57.5 \\
PM InDegree & 101.0 & 12.0 & 10.0 & 21.5 \\
PM OutDegree & 104.0 & 12.0 & 7.0 & 22.0 \\
Num Posts & 299.5 & 50.0 & 72.5 & 134.0 \\
Thread InDegree & 500.5 & 182.0 & 180.0 & 333.0 \\
Thread OutDegree & 527.0 & 183.5 & 198.0 & 332.0 \\
\hline Total Pairs & \multicolumn{3}{|c|}{314} & \multicolumn{3}{|c}{62} \\
\hline
\end{tabular}

Table 8: Statistics for ripper accusers and accusees. The numbers represent the medians across all accuser/accused pairs.

comprise over $10 \%$ of the bans. Rippers are individuals who rip off other members, and threads are created to identify these users.

\subsection{Accusers vs. Accused}

We next investigate the repercussions of accusing one member of being a ripper on the $\mathrm{CC}$ forum. To do this, we extracted all the threads where a user accused another member of being a ripper. The titles of these threads often take the form "Ripper < username>". Once we identified both parties, we compared the amount of activity the users engaged in prior to the accusation time. Table 8 shows the median values for several statistics about the accusers and the accused. We see that, in the cases where the accused person was ultimately banned, the accusers were much more active on the $\mathrm{CC}$ forum. For example, accusers had more than eight times the number of links in their PM graphs than the banned accused users, and the accusers had roughly twice as many links in their thread graphs. In the cases where the accusation did not result in a ban, the accused exhibited more activity than the accusers. Also, the unbanned accused users had a larger number of links in their PM (e.g., 10 vs. 21 for PM indegree) and thread (e.g., 180 vs. 330 for thread indegree) graphs than their banned counterparts.

\section{CONCLUSION}

This paper has characterized the social network makeup for six underground forums, how users interact, how baseline reputation is established and how it changes over time. This work is a first step in a larger research agenda to understand the social dynamics of the underground and how they impact e-crime market efficiencies.

\section{Acknowledgments}

We thank the reviewers for their valuable suggestions, and Vyas Sekar for his assistance with the accompanied public reviews and comments. This work was supported by NSF grants NSF-0433668 and NSF-0831138, ONR MURI grant N000140911081, and by generous support from Google, Microsoft, Yahoo, Cisco, and the UCSD Center for Networked Systems (CNS). McCoy was supported by a CCC-CRA-NSF Computing Innovation Fellowship. 


\section{REFERENCES}

[1] Ivision Power Board. http://www . invisionpower. com.

[2] vBulletin. http://www.vbulletin.com.

[3] H. Fallmann, G. Wondracek, and C. Platzer. Covertly Probing Underground Economy Marketplaces. In Proceedings of DIMVA, July 2010.

[4] J. Franklin, V. Paxson, A. Perrig, and S. Savage. An Inquiry into the Nature and Causes of the Wealth of Internet Miscreants. In Proceedings of CCS, October 2007.

[5] T. J. Holt and E. Lampke. Exploring Stolen Data Markets Online: Products and Market Forces. Criminal Justice Studies, 23(1):33-50, 2010.

[6] A. Mislove, M. Marcon, K. P. Gummadi, P. Druschel, and B. Bhattacharjee. Measurement and Analysis of Online Social Networks. In Proceedings of IMC, October 2007.
[7] J. Radianti. A Study of a Social Behavior inside the Online Black Markets. In Proceedings of SECURWARE, July 2010.

[8] A. Shilman. The Underground Credentials Market. Computer Fraud and Security, 2010(3):5-8, 2010.

[9] B. Stone-Gross, T. Holz, G. Stringhini, and G. Vigna. The Underground Economy of Spam: A Botmaster's Perspective of Coordinating Large-Scale Spam Campaigns. In Proceedings of LEET, 2011.

[10] R. Thomas and J. Martin. The Underground Economy: Priceless. ;login:, 31(6):7-16, Dec. 2006.

[11] C. Wilson, B. Boe, A. Sala, K. P. N. Puttaswamy, and B. Y. Zhao. User Interactions in Social Networks and their Implications. In Proceedings of EuroSys, April 2009.

[12] J. Zhuge, T. Holz, C. Song, J. Guo, X. Han, and W. Zou. Studying Malicious Websites and the Underground Economy on the Chinese Web. In Proceedings of WEIS, June 2008. 